\title{
Augmented Prototyping of 3D Rigid Curved Surfaces
}

\author{
Marina Atsumi Oikawa * Igor de Souza Almeida ${ }^{\dagger} \quad$ Takafumi Taketomi ${ }^{*} \quad$ Goshiro Yamamoto ${ }^{\S}$ \\ Jun Miyazaki ${ }^{\mathrm{I}} \quad$ Hirokazu Kato $\|$ \\ Graduate School of Information Science \\ Nara Institute of Science and Technology, Japan
}

\begin{abstract}
This paper presents an application of Augmented Reality (AR) in Rapid Prototyping (RP) of non-textured rigid curved surfaces. By enhancing the prototypes with AR, evaluation of its design and aesthetic concepts in real-time becomes easier, saving time and production costs. In our application, no fiducial markers are required and the CAD model used to build the prototype is applied in an edge-based tracking system specially designed to deal with curved shapes. Results from a pilot user study comparing the use of a 3D software and the proposed application are also presented.
\end{abstract}

Keywords: product design, augmented prototyping, model-based tracking, non-textured curved surfaces.

Index Terms: H.5.1 [Information Interfaces and Presentation]: Multimedia Information Systems-Artificial, augmented, and virtual realities; H.5.2 [Information Interfaces and Presentation]: User Interfaces-Prototyping

\section{INTRODUCTION}

During conception of a new product or design, in general some kind of model is created to attend as close as possible its requirements. Sketches, virtual models or rough physical models are some common ways used for this purpose. With RP, physical prototypes are automatically constructed using, for instance, a $3 \mathrm{D}$ printer.

However, RP is still time consuming, with some prototypes taking several hours to be finished. Furthermore, regarding evaluation of aesthetic features (such as color, texture and materials), since they are trial-and-error basis, RP may not be the ideal solution. Current $3 \mathrm{D}$ printers support these features, but making new prototypes only to evaluate them are not worth the time and cost, specially for complex shapes. In this sense, Augmented Prototyping (AP) represents a promising solution to overcome these shortcomings.

\section{Motivation and Related Work}

AP basically employs AR technology to combine virtual and physical prototypes [6]. Many approaches have been proposed, focusing on different aspects of AR. For instance, tangible AP is explored in [1] using foam mock-ups and in [3], the focus is on designing for handheld products using low-cost devices. However, both approaches require the use of fiducial markers to register the virtual objects. This brings some disadvantages such as: markers are intrusive elements and its partial occlusion may cause tracking failures; also, depending on the object shape or size, it is difficult to place the markers or they may occlude important parts of the prototype.

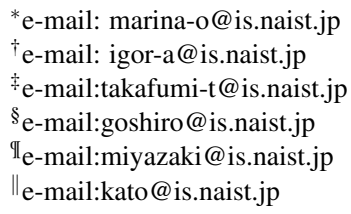

IEEE International Symposium on Mixed and Augmented Reality 2012 Science and Technology Proceedings

5 - 8 November 2012, Atlanta, Georgia

978-1-4673-4661-0/12/\$31.00 @2012 IEEE

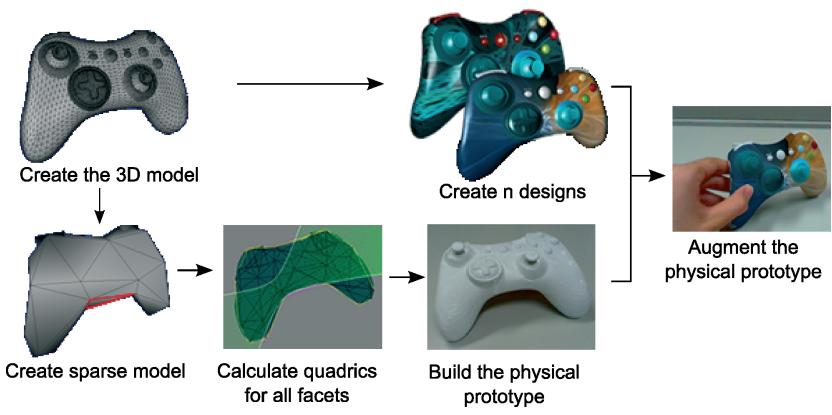

Figure 1: An overview of the proposed AP application.

Markerless AR has gained more attention, using features that can be naturally found in the object or in the environment to perform tracking instead of using fiducial markers. However, approaches relying only on the texture information found on the object surface may not be effective because the produced prototypes usually do not have enough distinctive features to be used for tracking.

In [4], prototype augmentation is achieved by using digital projectors to display the virtual information on the object surface (Spatial AR). In this case, head-mounted displays or handheld devices are not required and collaboration between the users becomes easier. However, some drawbacks are the shadows that are cast onto the objects by the user and problems with using camera based tracking where the object being tracked is also being projected onto.

The main contribution of this work is the application in AP of an edge-based tracking system specially designed to deal with curved shapes. No fiducial markers are needed and the CAD model used to build the prototype is used for matching with the edge information found on the video image. Then, different appearances of the target object can be created by the designer and overlaid on the prototype, facilitating visual comparison. A method to smoothly blend the augmented texture on the prototype was also developed as well as an approach to correct display the texture when it is occluded by the user's hand or other objects.

\section{IMPLEMENTATION DETAILS}

\subsection{Tracking Framework}

The algorithm used for tracking in this paper is the approach developed by [2]. In this approach, sparse polygonal meshes are suggested to solve the trade-off between computational time and tracking accuracy when dealing with curved surfaces. The vertex coordinates from the original dense mesh is used to calculate the parameters of an implicit model, represented by a general quadric equation. Those quadrics are attached to each mesh facet of the sparse mesh to recover the smoothness of the object and its projection represents the object apparent contour. During tracking, the distance between detected image edge points and corresponding points on the projected quadrics (conic curves) is minimized. The correspondences are established automatically and iteratively refined. An overview describing the system is given in Figure 1. 


\subsection{Texture composition algorithm and occlusion}

For rendering the object texture, OpenGL was used with a 2-path algorithm: in Path 1, the depth buffer of the target object is updated without updating the color buffer and in Path 2, if the Z-Buffer value is equal to the fragment $\mathrm{z}$ value, the color buffer is updated. The following equation is used for the texture blending:

$$
\text { out putColor }=\text { frameBufferColor } * \text { textureColor }
$$

This 2-path approach was adopted because the texture blending has to be applied only for the most front surface patches. If standard Z-buffer rendering is used, this texture blending would be applied for several patches which are not located on the most front surface, if they are rendered before the most front surface patches.

To correctly handle the occlusion of the texture, HSV (hue, saturation and value) was used for color segmentation. The prototype color was segmented in order to distinguish any kind of occlusion, such as by the user's hand or by another object. Each HSV channel was thresholded and combined into a mask used to distinguish the object shape from other elements in the scene.

\section{EXPERIMENTS}

A pilot user study comparing our proposed AP system against a 3D modeling software (Blender ${ }^{1}$ ) was designed to evaluate the perception of the user when visualizing different textures on a prototype. The objects used in our experiments were a prearranged non-textured small angel figurine and a game controller ${ }^{2}$, created using the $3 \mathrm{D}$ printer ZPrinter 450 .

A total of 10 students, 7 male and 3 female, ranging in age from 24 to 37 years old, participated in this study. 7 of the participants declared not having previous experience with any kind of 3D modeling software and only 1 participant never tried AR before.

Two conditions were tested: (a) using a 3D modeling software and (b) using the proposed AP system. For the first condition, a picture of the real environment was taken and placed in the $3 \mathrm{~d}$ environment as a static background. The participant could interact with the 3D model using mouse and keyboard. For the second condition, the keyboard was used only to press a key to change the object texture; all other interactions could be done by the users' hands.

In task 1, the users were asked to place the angel figurine in the desired position and choose among the available textures which one in their opinion best fitted with the other elements in the scene. In task 2, the users played the role of designers developing a new game controller skin based on a video game related to music and choose the best match with some posters in the background. Three textures were available for both prototypes and the users could browse among them as many times as they wanted (Figure 2).

\subsection{Results}

A within-subjects design was used with a single independent variable (3D modeling and AP application). A Likert scale (7-points, $1=$ Disagree, $7=$ Agree) was used for evaluation according to six measures: Ease of Use, Helpfulness, Ease of Comparison, Ease of Understanding, Precision and Ease of Visualization [5].

Significant differences between conditions were found for Ease of Use $(\mathrm{F}(1,36)=4.113, \mathrm{P}<0.05)$, Helpfulness $(\mathrm{F}(1,36)=4.113$, $\mathrm{P}<0.05)$, Ease of Comparison $(\mathrm{F}(1,36)=4.113, \mathrm{P}<0.05)$ and Precision $(\mathrm{F}(1,36)=4.113, \mathrm{P}<0.05)$. Significant differences between tasks were also found for Precision $(\mathrm{F}(1,36)=4.113, \mathrm{P}<0.05)$ and Ease of Visualization $(\mathrm{F}(1,36)=4.113, \mathrm{P}<0.05)$. Considering the average scores of the measures (Figure 3 ), the AP system consistently had higher rating for the aforementioned metrics. For the metric Ease of Understanding, no significant difference between conditions or tasks were found. In general, the results of this pilot user study showed good results and most of the users enjoyed the

${ }^{1}$ http://www.blender.org/, version 2.49

${ }^{2}$ http://www.blendswap.com/blends/electronics/xbox-360-controllers/

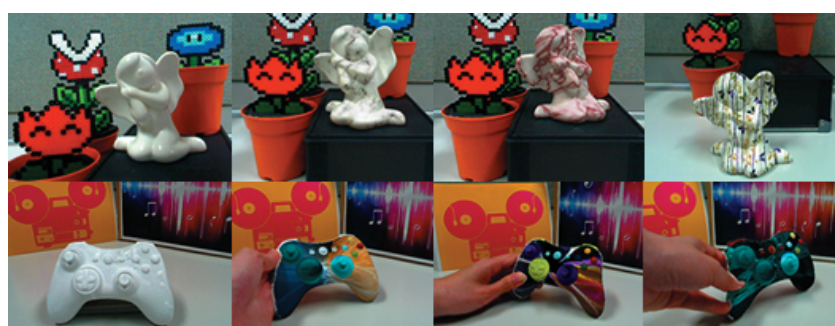

Figure 2: The objects used in the experiments without texture and with the three textures used for evaluation.

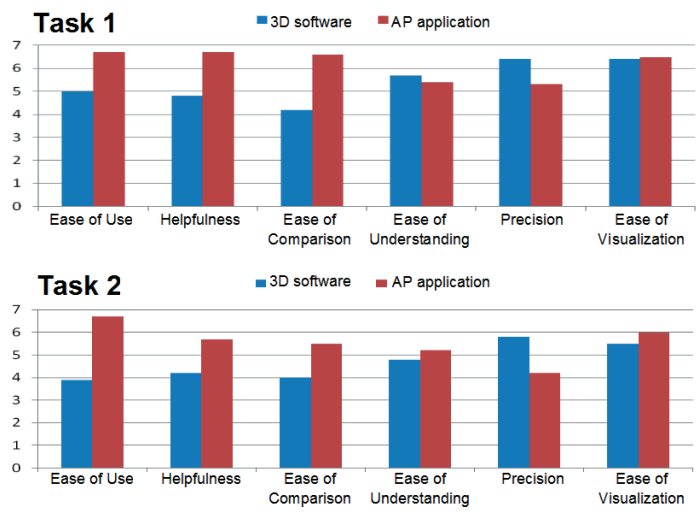

Figure 3: Average scores for the Likert scale measures.

experience. However, there are still some issues to be solved, most of them related to the object tracking, not the application itself. For instance, some users pointed out the jittering of the 3D model as distracting and the main problem with this approach. This explains the lower rating of the Precision metric.

\section{Conclusion}

This paper proposed an AP application to help validation of design solutions for rigid curved surfaces. Our method allows easy and fast evaluation of aesthetic features and does not require the use of markers or texture on the object surface. Hence, it is a suitable application for prototypes evaluation, since usually they are nontextured objects. The pilot user study gave us a first impression of the acceptance of this idea as well as improvement points in the tracking approach (jittering) and ideas for future experiments.

\section{REFERENCES}

[1] W. Lee and J. Park. Augmented foam: A tangible augmented reality for product design. In Proceedings of the International Symposium on Mixed and Augmented Reality, pages 106-109, 2005.

[2] M. A. Oikawa, T. Taketomi, G. Yamamoto, M. Fujisawa, T. Amano, J. Miyazaki, and H. Kato. Local quadrics surface approximation for real-time tracking of textureless 3d rigid curved objects. In Proceedings of the XIV Symposium on Virtual and Augmented Reality, 2012.

[3] H. Park, H. C. Moon, and J. Y. Lee. Tangible augmented prototyping of digital handheld products. Computers in Industry, 60:114-125, 2009.

[4] S. R. Porter, M. R. Marner, R. T. Smith, J. E. Zucco, and B. H. Thomas Spatial augmented reality for interactive rapid prototyping. In Proceedings of the 20th International Conference on Artificial Inteligence and Telexistence, pages 110-117, 2010.

[5] T. Tullis and B. Albert. Measuring the User Experience: Collecting, Analyzing and Presenting Usability Metrics. Morgan Kaufmann, 2008.

[6] J. Verlinden and I. Horvath. A critical systems position on augmented prototyping systems for industrial design. In Proceedings of the ASME 2007 International Design Engineering Technical Conferences and Computers and Information in Engineering Conference, 2007. 